\title{
INFLUÊNCIA DE IMPACTOS ANTRÓPICOS NA COMUNIDADE DE MACROINVERTEBRADOS NA BACIA DO BAIXO RIO GRANDE
}

\author{
Paulo Ricardo da Silva Camargo* \\ Fagner de Souza** \\ Patrícia Andressa de Almeida Buranello****
}

RESUMO: Os impactos antrópicos em ambientes aquáticos são os principais responsáveis pelas mudanças nas estruturas das comunidades aquáticas. Estes impactos podem ser causados, principalmente, pela instalação de barragens, degradação da mata ciliar e/ou despejo de resíduos domésticos e industriais, que alteram a dinâmica física, química e biológica dos ambientes naturais. Neste sentido, traçamos as hipóteses de que há diferenças nos parâmetros ecológicos entre comunidades de macroinvertebrados em ambientes com distintas proximidades da represa e em ambientes pré e pós-barragem. Desta maneira, objetivamos analisar os parâmetros ecológicos das comunidades de macroinvertebrados distribuídos em diferentes pontos no rio Uberaba (bacia do baixo rio Grande). Para isso, realizamos quatro coletas, em diferentes períodos sazonais, em quatro pontos com diferentes características hidrodinâmicas. Foram realizadas análises comparativas entre os pontos com base em dados de diferentes parâmetros ecológicos: abundância total, riqueza de espécies, diversidade de Shannon e Simpson, equitabilidade de Shannon e dominância de Simpson. Com base nos resultados, apenas na análise entre as regiões pré e pós-barragem foram encontradas diferenças significativas nos parâmetros dominância, diversidade de Shannon, diversidade de Simpson e equitabilidade. Essas diferenças não foram creditadas aos impactos endógenos da barragem e sim aos impactos antrópicos exógenos, como ausência de mata ciliar, atividades de pastoreio e despejo de resíduos domésticos.

PALAVRAS-CHAVE: Ambiente híbrido; Bacia do rio Paraná; Espécies bioindicadoras; Impacto ambiental; Parâmetros ecológicos.

Universidade Federal do Triângulo Mineiro, Instituto de Ciências Exatas, Naturais e Educação (ICENE), Brasil. E-mail: ricarduber@hotmail.com

** Universidade Estadual de Maringá, PPG em Ecologia de Ambientes Aquáticos Continentais PEA, Coleção Ictiológica do Nupélia, Brasil.

*** Universidade Federal do Triângulo Mineiro, Instituto de Ciências Exatas, Naturais e Educação (ICENE), Brasil. 


\title{
THE EFFECTS OF ANTHROPIC IMPACTS ON MACRO- INVERTEBRATE COMMUNIT Y IN THE LOWER BASIN OF THE RIO GRANDE
}

\begin{abstract}
The anthropic impacts on aquatic environments are the main causes in the structure of water communities. Impacts may be mainly caused by the construction of dams, degradation of riparian vegetation and launching of domestic and industrial wastes that change the physical, chemical and biological dynamics of natural environments. A hypothesis is raised that there are differences in ecological parameters between macro-invertebrate communities in sites with different distance from dams and in pre- and post-dam environments. Ecological parameters of macro-invertebrate communities distributed at different site of the river Uberaba (basin of the lower river Grande) are investigated. Four collections were undertaken at different seasons, in four sites, with different hydro-dynamic features. The sites were compared by different ecological parameters, such as total abundance, species richness, Shannon and Simpson diversity, Shannon equitability and Simpson dominance. Results show that there were significant differences in the parameters dominance, Shannon diversity, Simpson diversity and equitability at the sites between pre- and post-dam regions. Differences were credited on exogenous anthropic impacts, such as riparian vegetation, pasturing and launching of domestic wastes, rather than on endogenous impacts of the dam.
\end{abstract}

KEY WORDS: Hybrid environment; River Paraná basin; Bio-indicating species; Environmental impact; Ecological parameters.

\section{INTRODUÇÃO}

Os fortes impactos antrópicos em ambientes lóticos vêm sendo, nas últimas décadas, um dos principais fatores negativos na desestruturação das comunidades aquáticas (THORNE; WILLIAMS, 1997; MCALLISTER; HAMILTON; HARVEY, 1997; CALLISTO; MORENO; BARBOSA, 2001; RODRIGUES; MALAFAIA; CASTRO, 2010; SOUZA et al., 2015). Estes impactos podem ser causados pela alteração do fluxo hídrico (AGOSTINHO; JÚLIO JR.; BORGHETTI, 1992), escoamento de resíduos industriais, domésticos e agropecuários (SHEPP; CUMMINS, 1997; CUNICO et al., 2012), e introdução de espécies exóticas (CUNICO; AGOSTINHO; LATINI, 2006; SOUZA; TOZZO, 2013). 
Outro fator muito comum nos ambientes aquáticos brasileiros são os represamentos, que estão presentes em rios de diferentes ordens, no qual o rompimento do fluxo longitudinal causa diversas alterações estruturais abióticas (WARD; STANFORD, 1983) e bióticas (AGOSTINHO; JÚLIO JR; BORGHETTI, 1992). As alterações abióticas podem ser: transformação em um ambiente de características híbridas; sedimentação de matéria orgânica; diminuição da concentração de oxigênio e aumento de respiração anaeróbica; aumento da transparência da água; aumento da produção primária fitoplanctônica; dentre outras. As alterações bióticas podem ser: imposição de barreira física, impedindo deslocamento de migradores; diminuição da taxa de reprodução; menor eficiência fisiológica por ausência de oxigênio; mudança na dieta das espécies; aumento da abundância e dominância de espécies mais resistentes; facilitação para invasão e estabilização de espécies exóticas; dentre outras (AGOSTINHO; JÚLIO JR; BORGHETTI, 1992).

Muitos autores discutem sobre como a degradação e a qualidade ambiental influenciam na estrutura ecológica das comunidades (RODRIGUEZ; LEWIS, 1994; KELLY; WHITTON, 1998; MUSTOW, 2002; USSEGLIO-POLATERA et al., 2000; LUIZ et al., 2005; SOUZA et al., 2013; SOUZA et al., 2014; SOUZA et al., 2015; MISE et al., 2015). Desta forma, a estrutura da comunidade, avaliada por meio dos parâmetros ecológicos, pode revelar a qualidade ambiental (LUIZ et al., 2005; YOSHIDA; ROLLA, 2012), assim como inferir sobre as interações (positivas ou negativas) que podem ocorrer no ambiente analisado (HELMS; HELMS; FEMINELLA, 2009; FOGAÇA; GOMES; HIGUTI, 2013).

Com esse intuito, técnicas como monitoramento têm sido desenvolvidas e aplicadas constantemente para evidenciar impactos em ambientes aquáticos, dentre estas se destacam técnicas observacionais (CALLISTO et al., 2002) e de monitoramento bióticos, principalmente com o uso de macroinvertebrados aquáticos (LOPES et al., 2011).

Estes organismos são conhecidos como invertebrados pertencentes a vários grupos taxonômicos, visíveis a olho nu e que habitam o ambiente aquático, especialmente a região bentônica, no mínimo em alguma etapa do seu ciclo de vida. Seus principais representantes são os Anelídeos, Moluscos, Crustáceos e Insetos (MUGNAI; NESSIMIAN; BAPTISTA, 2010). A capacidade destes grupos de habitar 
diversos tipos de ambientes aquáticos ajuda no diagnóstico do estado qualitativo ambiental, uma vez que podem refletir as consequências negativas na estruturação ecológica de sua comunidade (TATE; HEINY, 1995; LOPES et al., 2011).

A eficácia dos macroinvertebrados aquáticos como bioindicadores se dá devido aos seguintes fatores: 1) são sensíveis a diferentes impactos e reagem rapidamente de forma gradual; 2) não realizam grandes migrações, são ótimos representantes de condições locais; 3) apresentam grande abundância; 4) são fáceis de serem coletados e identificados em altos níveis taxonômicos (ordem e família); 5) a comunidade é diversificada, apresentando um grande número de táxons e níveis tróficos; 6) exibem um ciclo de vida relativamente curto, respondendo de forma rápida às alterações ambientais por meio de sua estrutura ecológica (LOPES et al., 2011).

Além disso, alguns grupos de macroinvertebrados aquáticos são relatados como sensíveis/tolerantes e bons bioindicadores de ambientes naturais, como Ephemeroptera, Plecoptera e Trichoptera (FOGAÇA; GOMES; HIGUTI, 2013), e/ou impactados, como Chironomidae, Hydrophilidae e Gerridae (MARQUES; BARBOSA; CALLISTO, 1999). Estes fatos, junto com a verificação da estrutura ecológica, já se demonstraram eficientes para evidenciar a qualidade ambiental, como, por exemplo, nos trabalhos de Bieger et al. (2010), Yoshida e Rolla (2012), e Souza et al. (2014).

Com base na discussão exposta e visando a aplicação em um ambiente híbrido, traçamos as seguintes hipóteses: 1) há diferenças nos parâmetros ecológicos das comunidades de macroinvertebrados em ambientes com distintas proximidades da represa; e 2) ambientes pré e pós-barragem exibem diferenças em seus parâmetros ecológicos. Nossa premissa se pauta no conceito de descontinuidade seriada (WARD; STANFORD, 1983) e domínio de processos (MONTGOMERY, 1999), estes autores argumentam que o rio apresenta manchas estruturais que são regidas por processos locais e a sua relação tetradimensional, e não apenas longitudinal. Desta forma, objetivamos analisar a estrutura ecológica da comunidade de macroinvertebrados do rio Uberaba, submetidas a diferentes pressões e regimes hidrológicos. 


\section{MATERIAL E MÉTODOS}

\section{1 ÁREA DE ESTUDO}

Foram amostrados quatro trechos no percurso longitudinal do rio Uberaba (pontos A-D) no município de Uberaba (MG), cada trecho medindo 100 metros, e estes foram divididos em dois grupos (pré-barragem e pós-barragem) (Figura 1):

Pré-barragem: foram determinados dois trechos antes da barragem de captação de água da cidade, denominada como "barragem da Codau" (ponto A e B): Ponto A: equivale a montante deste trecho; localizada na zona rural nas coordenadas $19^{\circ} 38^{\prime} 26,77^{\prime}$ 'S $/ 47^{\circ} 53$ '12,70'O. De acordo com Souza et al. (2016), esse ambiente apresenta matas ciliares densas em ambos lados, o leito é composto com quase nada de sedimento fino por rochas de tamanhos distintos; o fluxo hídrico se alterna em corredeiras e poços. Ponto B: é a região de jusante, bem próxima a barragem, localizada em uma região com características de zona rural, nas coordenadas $19^{\circ} 42^{\prime} 48,35^{\prime \prime} \mathrm{S} / 47^{\circ} 56^{\prime} 17,94^{\prime \prime} \mathrm{O}$. Ainda de acordo com o mesmo autor, a margem direita possui uma mata ciliar densa, já em sua margem esquerda exibe regiões desprovidas de mata e outras cobertas por gramíneas, seu leito é composto por sedimento fino, com poucas rochas; seu fluxo é predominante lêntico.

Pós-barragem: foram determinados dois trechos abaixo da barragem de captação de água (pontos $\mathrm{C}$ e D). Ponto C: é o trecho mais próximo a barragem; situado em uma região bem próxima do perímetro urbano de Uberaba, mas com características de regiões de zona rural, como no estabelecimento de atividades de agropecuária, nas coordenadas $19^{\circ} 43^{\prime} 17,28$ 'S/47 $56^{\prime} 51,95$ ”O. Os autores Souza et al. (2016) assumem que seu leito é composto por rochas de grande porte, o leito apresenta poucas regiões de sedimento fino, o fluxo hídrico se alterna entre corredeiras e poços fundos (em relação ao ponto A). Ponto D: é o trecho mais a jusante, está localizado no perímetro urbano de Uberaba no distrito Alfredo Freire, próximo a central de tratamento de esgoto, nas coordenadas $19^{\circ} 433^{\prime} 45,18$ 'S $/ 47^{\circ} 59^{\prime} 55,57^{\prime \prime} \mathrm{O}$. Conforme Souza et al. (2016), esse ponto possui uma densa mata ciliar em ambas as margens; distintos tamanhos de rochas e seixos compõem o seu leito, seu fluxo na maior parte é de corredeiras, mas apresenta alguns remansos próximos a sua margem. 


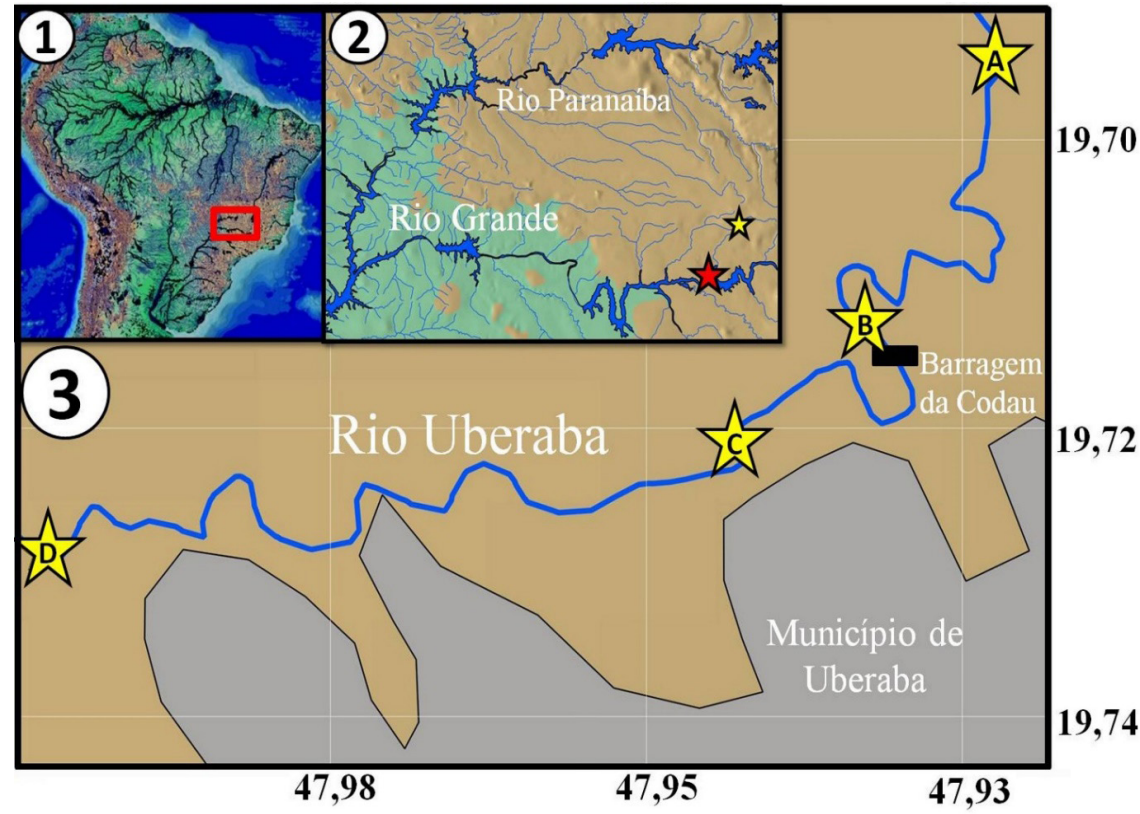

Figura 1. Área de estudo. 1) localização na bacia do alto rio Paraná; 2) localização na bacia do baixo rio Grande (estrela amarela = rio Uberaba; estrela vermelha = rio Grande); 3) localização dos pontos amostrados (A-D) no município de Uberaba (MG).

\subsection{AMOSTRAGEM}

As coletas foram realizadas em quatro campanhas, de outubro de 2014 a julho de 2015 (com intervalo de três meses entre as campanhas), para minimizar os efeitos ligados à sazonalidade. Os macroinvertebrados foram coletados com peneiras, de $1,0 \times 0,50 \mathrm{~m}$ com malha de $3 \mathrm{~mm}$ entre nós adjacentes, com esforço amostral de 50 minutos em cada ponto. Após coletados, foram fixados com formalina 10\% e conservados em álcool 70\%. Em laboratório foram identificados de acordo com Costa, Ide e Simonka (2006) e Mugnai, Nessimian e Baptista (2010). Para confirmar a eficiência amostral foi realizada uma curva do coletor com os dados de cada trecho amostrado. Para estas análises foi utilizado o software PAST versão 2.03 (HAMMER et al., 2001). 


\subsection{ANÁLISE DE DADOS}

Foram analisados espacialmente os seguintes parâmetros ecológicos: abundância total; riqueza de espécies; diversidade de espécies de Shannon e Simpson, equitabilidade de Shannon e dominância de Simpson. Todos os índices foram calculados no software PAST ${ }^{\circledR}$ (HAMMER et al., 2001). Após o cálculo dos parâmetros foram realizadas comparações espaciais de cada parâmetro ecológico. As análises foram realizadas em dois blocos analíticos: no bloco 1 foram realizadas as comparações espaciais entre os trechos pré-barragem (pontos A e B) versus pós-barragem (pontos $\mathrm{C}$ e D); no bloco 2 foram realizadas análises comparativas espaciais entre todos os pontos amostrados (pontos A-D). Para verificar as diferenças entre os pontos foi realizado o teste não-paramétrico de Kruskal-Wallis, pois os dados não atingiram os pressupostos de normalidade (teste de Shapiro-Wilk) e homocedasticidade (teste de Levine) necessários para testes paramétricos. Estas análises foram realizadas no software Statistica7 (STATSOFT, 2005).

\section{RESULTADOS}

Foram coletados 6.810 indivíduos, distribuídos em três filos, 12 ordens e 48 famílias. As famílias mais abundantes foram Chironomidae, com 2.394 indivíduos (35,2\%), Culicidae, com 1.565 indivíduos (23\%), e Ephydridae com 943 indivíduos (13,4\%). Já as que apresentaram menor quantidade de indivíduos foram Erpobdellidae (representantes do filo Annelida), Dolichopodidae, Phoridae, Simulidae, Stratiomyidae, Tabanidae, Veliidae, Gripopterygidae, Perlidae e Hydroosychidae (representantes do filo Arthropoda), todos com apenas 1 indivíduo $(0,01 \%)$ (Tabela 1$)$.

Em relação à abundância dos filos, Arthropoda apresentou 5.801 indivíduos (85,2\%), enquanto Mollusca apresentou 690 indivíduos (10,1\%) e Annelida 319 indivíduos (4,7\%). Já para a quantidade de indivíduos distribuídos entre as ordens, as com maior abundância foram Diptera, com 5.091 espécimes (74,8\%), Gastropoda, com 690 (10,1\%), Odonata, com 493 (7,3\%), e Hhynchobdellida, com 
209 (3,1\%). Com a menor abundância estão Tubificida, com 2 indivíduos (0,03\%), e Arnchynchobdellidae, com 1 indivíduo (0,01\%) (Tabela 1).

Com base na Figura 2, no cálculo curva do coletor, nota-se uma tendência da curva em assumir uma assíntota. Assim, podemos considerar que as coletas realizadas no rio Uberaba atingiram uma eficiência amostral aceitável para os objetivos do trabalho. Nas análises comparativas dos parâmetros ecológicos no bloco 1 (pré e pós-barragem), houve diferenças significativas em quatro parâmetros, dominância ( $p=0,0209)$, diversidade de Shannon $(\mathrm{p}=0,0209)$, diversidade de Simpson $(\mathrm{p}=0,0209)$ e equitabilidade $(\mathrm{p}=0,0209)$ (Figura 3). Já os parâmetros riqueza e abundância total não exibiram diferenças significativas (respectivamente, $p=0,0833$ e $p=0,3865$ ). Já nas análises comparativas do bloco 2 (relação entre os pontos A-D), não foi encontrada diferença significativa em nenhum dos parâmetros analisados (riqueza, $\mathrm{p}=0,8939$; abundância, $\mathrm{p}=0,1149$; dominância, $\mathrm{p}=0,1264$; diversidade de Shannon, $\mathrm{p}=0,1043$; diversidade de Simpson, $\mathrm{p}=0,1264$; $\mathrm{e}$ equitabilidade, $\mathrm{p}=0,1365)$.

Tabela 1. Lista de macroinvertebrados coletados no rio Uberaba, bacia do baixo rio Grande, distribuídos por pontos amostrados e total geral de cada táxon. Classificações seguiram Mugnai, Nessimian e Baptista (2010)

(Continua)

\begin{tabular}{llllll}
\hline Táxons & Ponto A & Ponto B & Ponto C & Ponto D & Total \\
\hline ANNELIDA & & & & & \\
Arnchynchobdellidae & & & & & \\
Erpobdellidae & - & - & - & 1 & 1 \\
Hhynchobdellida & & & & & \\
Glossiphoniidae & - & - & 31 & 29 & 60 \\
Hirundinae & 2 & - & - & & 2 \\
Pisicolidade & - & - & 15 & 132 & 147 \\
Oligochaeta & & & & & \\
$\begin{array}{l}\text { Oligochaetassp } \\
\text { Tubificida }\end{array}$ & - & - & - & 107 & 107 \\
$\begin{array}{l}\text { Naididae } \\
\text { ARTHROPODA }\end{array}$ & - & - & & & \\
Coleoptera & & & & & \\
\hline
\end{tabular}


(Continua)

\begin{tabular}{llllll}
\hline Táxons & Ponto A & Ponto B & Ponto C & Ponto D & Total \\
\hline Dityscidae & 1 & - & 2 & - & 3 \\
\hline Gyrinidae & 3 & - & 5 & - & 8 \\
Hidrophilidae & 55 & - & 3 & 14 & 72 \\
Staphylinidae & 1 & - & 2 & - & 3
\end{tabular}

\section{Diptera}

Ceratopogonidae

Chironomidae

Culicidae

Dolichopodidae

Empididae

Ephydridae

Muscidae

Phoridae spp.

Psycholidae

Sarcophagidae

Simulidae

Stratiomyidae

Tabanidae

Tipulidae

\section{Ephemeroptera}

Baetidae 6

Leptohyphidae

Leptophlebiidae

$\begin{array}{llll}- & - & 5 & 5 \\ 37 & 540 & 1816 & 2394 \\ - & 1 & 1564 & 1565 \\ - & - & 1 & 1 \\ - & - & 2 & 2 \\ - & 1 & 912 & 913 \\ - & - & 5 & 5 \\ - & - & 1 & 1 \\ - & - & 193 & 193 \\ - & 2 & - & 2 \\ - & - & 1 & 1 \\ - & - & 1 & 1 \\ - & - & 1 & 1 \\ 1 & 3 & 3 & 7\end{array}$

Hemiptera

Belostomatidae

3

31

10

44

Coreidae

19

1

Corixidae

4

Gerridae

Mesoveliidae

Naucoridae

4

Nepidae

913 
(Conclusão)

\begin{tabular}{|c|c|c|c|c|c|}
\hline Táxons & Ponto A & Ponto B & Ponto C & Ponto D & Total \\
\hline Veliidae & - & - & 1 & - & 1 \\
\hline Pleidae & 1 & - & - & 1 & 2 \\
\hline \multicolumn{6}{|l|}{ Odonata } \\
\hline Calopterygidae & 14 & 25 & 187 & 1 & 227 \\
\hline Dicteriadidae & 3 & 18 & 2 & - & 23 \\
\hline Gomphidae & 16 & 5 & 12 & - & 33 \\
\hline Libellulidae & 11 & 7 & 31 & 1 & 50 \\
\hline Protoneuridae & - & 59 & 2 & - & 61 \\
\hline Coenagrionidae & - & 94 & 8 & - & 102 \\
\hline \multicolumn{6}{|l|}{ Plecoptera } \\
\hline Gripopterygidae & 1 & - & - & - & 1 \\
\hline Perlidae & 1 & - & - & - & 1 \\
\hline \multicolumn{6}{|l|}{ Trichoptera } \\
\hline Calamoceratidae & 1 & 2 & - & - & 3 \\
\hline Hydroosychidae & 1 & - & - & - & 1 \\
\hline \multicolumn{6}{|l|}{ MOLLUSCA } \\
\hline \multicolumn{6}{|l|}{ Gastropoda } \\
\hline Physidae & 2 & 16 & 51 & 514 & 583 \\
\hline Planorbidae & - & 7 & 61 & 39 & 107 \\
\hline
\end{tabular}




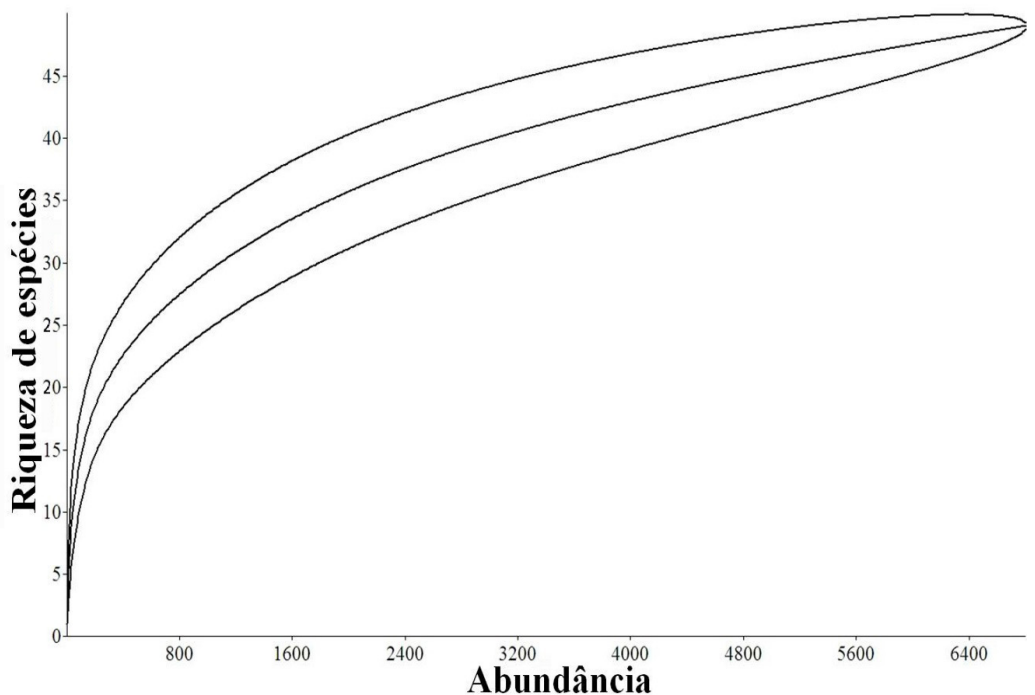

Figura 2. Curva do coletor para as amostragens realizadas no rio Uberaba, bacia do baixo rio Grande. Linha mediana = média, linhas periféricas $=$ desvio padrão.
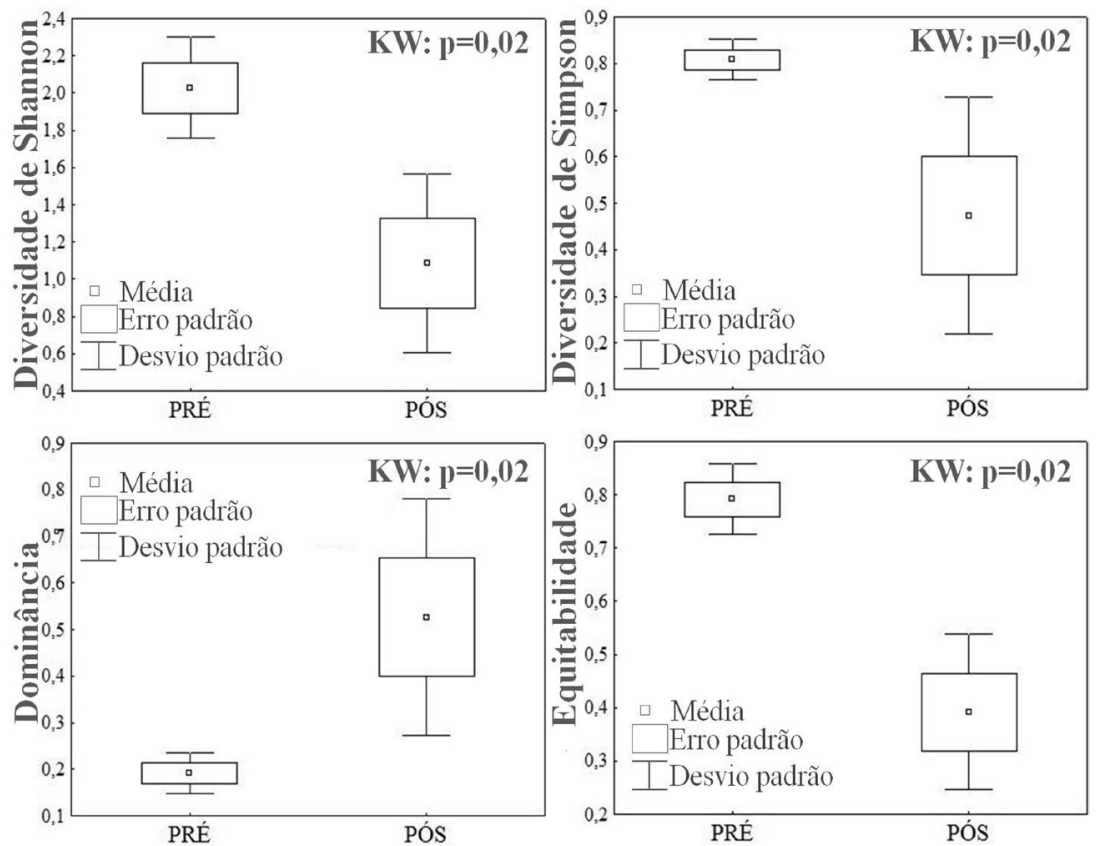

Figura 3. Análise espacial comparativa de Kruskal-Wallis entre os pontos pré (A e B) e pós-barragem (C e D) amostrados no rio Uberaba, bacia do baixo rio Grande. 


\section{DISCUSSÃO}

Nos resultados obtidos na relação pré e pós-barragem (bloco 2) a região abaixo da barragem apresentou menores valores nos parâmetros ecológicos, corroborando parcialmente com nossa hipótese inicial, mais especificamente a hipótese 2, de que há diferenças estruturais ecológicas nas comunidades de macroinvertebrados em ambientes com distintas posições em relação à represa. Esta divergência pode estar ligada com as características abióticas destes trechos, já que de acordo com Agostinho, Júlio Jr e Borghetti (1992) na região a jusante de uma barragem (região pós-barragem) há uma diminuição na disponibilidade de recursos e alterações drásticas nos parâmetros físicos e químicos, gerados pela retenção de matéria orgânica fragmentada, particulada e dissolvida na região a montante (prébarragem). Estes recursos, de acordo com Esteves (1998), estão diretamente ligados ao sucesso de colonização de macroinvertebrados. Assim, a ausência destes recursos pode ter afetado negativamente a estrutura da comunidade pós-barragem.

Além disso, nesta região (pós-barragem), localizada dentro do perímetro urbano (ver área de estudo), há ausência de mata ciliar em vários trechos e a presença de áreas de pastagem, pontos de lixiviação e aparentes despejos de resíduos domésticos. Estas ações podem interferir diretamente nos aspectos tróficos e físicoquímicos da água. Assim, recursos tróficos alóctones, oriundos da mata ciliar, não estão disponíveis para a estabilidade das redes tróficas. Desta maneira, de acordo com Elton (1958), a instabilidade de uma rede trófica prejudica a biodiversidade local, reduzindo assim a estabilização das comunidades e consequentemente a sua estrutura.

Neste mesmo sentido, os impactos externos aumentam as relações ecológicas não harmônicas, como exemplo a competição, no qual espécies mais adaptadas e/ ou mais competitivas sobressaem alterando a dinâmica ecológica da comunidade. Este fato pode ser observado na abundância expressada por Chironomidae (Tabela 1). Por esta família ser resistente e se adaptar a vários impactos (HAMADA et al., 
2014), como, por exemplo, baixas taxas de oxigênio (RIBEIRO; UIEDA, 2005), e ser generalista, ter rápida reprodução e alta taxa de dispersão (ARMITAGE; CRANSTON; PINDER, 1995; CALLISTO; ESTEVES, 1998; GOULARTE; CALLISTO, 2003; ALBURAYA; CALLIL, 2007; FAILLA et al., 2015) ela pode expressar uma maior vantagem adaptativa, em relação a outros organismos mais sensíveis, favorecendo sua colonização em ambientes impactados.

Além disso, o aumento de matéria orgânica, oriundo de ações antrópicas na margem, tem uma relação negativa com a estrutura da comunidade e favorece o sucesso de organismos mais resistentes (BUSS et al., 2002). Este evento é similar aos ocorridos na região pós-barragem, na qual há presença de intensas atividades antrópicas como lançamento de resíduos domésticos aparente, área ativa de pecuária e a ausência de mata ciliar. Assim, este impacto se amplifica em períodos chuvosos (observação pessoal), pois na ausência da retenção natural da matéria alóctone, propiciada pelas raízes da mata ciliar, há lixiviação do solo, levando todos os resíduos para o rio, causando erosão da margem e assoreamento do leito do rio. Fatos que favorecem aos organismos resistentes, como os da família Chironomidae (já discutidos anteriormente).

Outro ponto é que a qualidade ambiental está fortemente ligada aos fatores que contribuem tanto positivamente quanto negativamente para os parâmetros ecológicos de uma comunidade de macroinvertebrados, afetando a biodiversidade e a disponibilidade de nichos ecológicos (JESUS; CAVALHEIRO, 2004; SOUZA et al., 2014). Estes fatos são evidenciados pelos parâmetros que exibiram diferenças e significativas e pela abundância de alguns organismos bioindicadores. Apesar da abundância não ter diferenças significativas, este parâmetro tem uma relação direta com o cálculo dos outros parâmetros que exibiram diferenças significativas (ODUM, 1988; HAMMER; HARPER; RYAN, 2001).

Em relação ao ambiente pré-barragem, a presença de uma densa mata ciliar e poucos impactos externos de origem antropogênica (observação pessoal), possibilita a estruturação de um ambiente mais favorável, em relação ao ambiente 
pós-barragem, para vários grupos de macroinvertebrados, principalmente para as famílias de Odonata. Os representantes desta ordem necessitam de ambientes com maior complexidade ambiental mediada principalmente pela presença de mata ciliar (CARVALHO et al., 2013), que é utilizada (quando submersa ou fragmentada no leito do rio) como principal substrato na obtenção de recursos alimentares e de proteção (ASSIS; CARVALHO; NESSIMIAN, 2004). Fatos que não ocorrem nas regiões pósbarragem, devido suas características e influências de suas margens.

\section{CONSIDERAÇÕES FINAIS}

Em síntese, verificou-se que houve diferenças na estrutura das comunidades de macroinvertebrados entre as regiões pré e pós-barragem, corroborando parcialmente com a nossa hipótese inicial. Além disso, os impactos antrópicos alóctones, como degradação da mata ciliar, despejos de resíduos domésticos e atividade de pastoreio foram os principais fatores nas divergências estruturais entre as regiões, sendo estes aparentemente mais relevantes que os impactos hidrodinâmicos recorrentes da barragem. Portanto, é indispensável que ações mitigatórias sejam realizadas, como recomposição da mata ciliar, reparação e manutenção das galerias de saneamento básico (esgoto). Também é essencial uma fiscalização mais rígida sobre as atividades desenvolvidas nestes trechos e ações que visam desenvolver programas de educação ambiental que orientem a população sobre os impactos gerados neste ambiente, tanto no uso como nas atividades não condizentes ao bemestar ambiental. 


\section{AGRADECIMENTOS}

Ao laboratório de "ecologia aquática" da UFTM pelo suporte estrutural e de materiais. Ao Professor Doutor Paulo Fiuza (UFV) pelo empréstimo da Lupa. Aos graduandos Kevini, Michael, Manoela, Pedro Peixoto e Janaína Giagio pelo auxílio nas coletas e a Ingrid Marques e Luciano Fiuza pelo auxílio na triagem e identificação do material. F. Souza agradece ao Programa de Pós-Graduação em Ecologia de Ambientes Aquáticos Continentais (PEA/UEM) e à Coordenação de Aperfeiçoamento de Pessoal de Nível Superior (CAPES), pelo provimento de bolsa.

\section{REFERÊNCIAS}

AGOSTINHO, A. A.; JÚLIO JR., H. F.; BORGHETTI, J. R. Considerações sobre os impactos dos represamentos na ictiofauna e medidas para sua atenuação. Um estudo de caso: reservatório de Itaipu. Revista Unimar, v. 14, p. 89-107, 1992.

ALBURAYA, F. H.; CALLIL, C. T. Variação temporal de larvas de Chironomidae (Dipetra) no Alto rio Paraguai (Cáceres, Mato Grosso Brasil). Revista Brasileira de Zoologia, v. 24, n. 3, p. 565-572, 2007.

ARMITAGE, P. D.; CRANSTON, P. S.; PINDER, L. C. V. The Chironomidae: biology and ecology of non-biting midges. London: Chapman and Hall, 1995.

ASSIS, J. C. F. de; CARVALHO, A. L.; NESSIMIAN, J. L. Composição e preferência por microhábitat de imaturos de Odonata (Insecta) em um trecho de baixada do Rio Ubatiba, Maricá-RJ, Brasil. Revista Brasileira de Entomologia, v. 48, n. 2, p. 273282, 2004.

BIEGER, L.; CARVALHO, A. B. P.; STRIEDER, M. N.; MALTCHIK, L.; STENERT, C. Are the streams of the Sinos River basin of good water quality? Aquatic macroinvertebrates may answer the question. Brazilian Journal of Biology, v. 70, n. 4, p. 1207-1215, 2010.

BUSS, D. F.; BAPTISTA, D. F.; SILVEIRA, M. P.; NESSIMIAN, J. L.; DORVILLÉ, L. F. M. 
Influence of water chemistry and environmental degradation on macroinvertebrate assemblages in a river basin in south-east Brazil. Hydrobiologia, v. 81, p. 125-136, 2002.

CALliSTO, M.; FERREIRA, W. R.; MORENO, P.; GOULART, M.; PETRUCIO, M. Aplicação de um protocolo de avaliação rápida da diversidade de habitats em atividades de ensino e pesquisa (MG-RJ). Acta Limnologica Brasiliensia, v. 14, n. 1, p. 91-98, 2002.

CALLISTO, M.; ESTEVES, F. A. Biomonitoramento da macrofauna bentônica de Chironomidae (Diptera) em dois igarapés amazônicos sob influência das atividades de uma mineração de bauxite. In: NESSIMIAN, J. L.; CARVALHO, A. L. (Eds). Ecologia de insetos aquáticos. Rio de Janeiro, PPGE-UFRJ, Série Oecologia Brasiliensis, 1998. p. 299-309.

CALliSTO, M.; MORENO, P.; BARBOSA, F. A. R. Habitat diversity and benthic functional trophic groups at Serra do Cipó, Southeast Brazil, Revista Brasileira de Biologia, v. 61, n. 2, p. 259-256, 2001.

CARVALHO, F. G.; SILVA-PINTO, N.; OLIVEIRA-JÚNIOR, J. M. B.; JUEN, L. Effects of marginal vegetation removal on Odonata communities. Acta Limnologica Brasiliensia, v. 25, n. 1, p. 10-18, 2013.

COSTA, C.; IDE, S.; SIMONKA, C. E. Insetos imaturos: metamorfose e identificação. Ribeirão Preto: Holos, 2006.

CUNICO, A. M.; AGOSTINHO, A. A.; LATINI, J. D. Influência da urbanização sobre as assembléias de peixes em três córregos de Maringá, Paraná. Revista Brasileira de Zoologia, v. 23, n. 4, p. 1101-1110, 2006.

CUNICO, A. M.; FERREIRA, E. A.; AGOSTINHO, A. A.; BEAUMORD, A. C.; FERNANDES, R. The effects of local and regional environmental factors on the structure of fish assemblages in the Pirapó Basin, Southern Brazil. Landscape and Urban Planning, v. 105, n. 3, p. 336-344, 2012.

ELTON, C. S. The Ecology of Invasions by Animals and Plants. London: Methuen, 1958. 
ESTEVES, F. de A. Fundamentos de limnologia. $2^{\mathrm{a}}$ ed. Rio de Janeiro: Editora Interciência, 1998.

FAILLA, A. J.; VASQUEZ, A. A.; FUJIMOTO, M.; RAM, J. L. The ecological, economic and public health impacts of nuisance chironomids and ther potential as aquatic invaders. Aquatic Ivasions, v. 10, n. 1, p. 1-15, 2015.

FOGAÇA, F. N. O.; GOMES, L. C.; HIGUTI, J. Percentage of Impervious Surface Soil as Indicator of UrbanizationImpacts in Neotropical Aquatic Insects. Neotropical Entomology, v. 42, n. 5, p. 483-491, 2013.

GOULART, M.; CALLISTO, M. Bioindicadores de qualidade de água como ferramenta em estudo de impacto ambiental. Revista da FAPAN, v. 2, n. 1, p. 156-164, 2003.

HAMADA, N; NESSIMIAN, J. L.; QUERINO, R. B. Insetos aquáticos na Amazônia brasileira: taxonomia, biologia e ecologia. Manaus: Editora do INPA, 2014.

HAMMER, O.; HARPER, D. A. T.; RYAN, P. D. PAST: Paleontological Statistic software package for education and data analysis. Palaeontologia Electronica, Oslo, v. 4, n. 1, p. 9, 2001.

HELMS, B. S.; HELMS, J. E.; FEMINELLA, W. Seasonal variability of landuse impacts on macroinvertebrate assemblages in streams of western Georgia, USA. Journal of the North American Benthological Society, v. 28, n. 4, p. 991-1006, 2009.

JESUS, N.; CAVALHEIRO, F. Aspectos antrópicos, legais e conservacionistas na Serra do Japi, SP. In: SANTOS, J. E. (Org.). Faces da polissemia da paisagem: ecologia, planejamento e percepção. São Carlos: Rima, 2004. v. 2.

KELLY, M. G.; WHITTON, B. A. Biological monitoring of eutrophication in Rivers. Hydrobiologia, v. 384, n. 3, p. 55-67, 1998.

LOPES, A.; PAULA, J.; MARDEGAN, S. F.; HAMADA, N.; PIEDADE, M. T. F. Influência do habitat na estrutura da comunidade de macroinvertebrados aquáticos associados às raízes de Eichhorniacrassipes na região do Lago Catalão, Amazonas, Brasil. Acta Amazônica, v. 41, n. 4, p. 493-502, 2011.

LUIZ, E. A.; PETRY, A. C.; PAVANELLI, C. S.; JÚNIOR, J.; HORACIO FERREIRA, L.; 
DIRÇO, J.; DOMINGUES, V. M. As assembléias de peixes de reservatórios hidrelétricos do estado do Paraná e bacias limítrofes. In: RODRIGUES, L.; THOMAZ, S. M.; AGOSTINHO, A. A.; GOMES, L. C. (Ed.). Biocenoses em reservatórios padrões espaciais e temporais. São Carlos: Ed. Rima, p. 107-113, 2005.

MARQUES, M. M. G. S. M.; BARBOSA, F. A. R.; CALLISTO, M. Distribution and abundance of Chironomidae (Diptera, Insecta) in an impacted watershed in southeast Brazil. Brazilian Journal of Biology, v. 59, n. 4, p. 553-561, 1999.

MCALLISTER, D. E.; HAMILTON, A. L.; HARVEY, B. Global freshwater biodiversity: striving for the integrity of freshwater ecosystems. Sea Wind, v. 11, n. 3, p. 1-142, 1997.

MISE, F. T.; SOUZA, F.; PAGOTTO, J. P. A.; GOULART, E. Intraspecific ecomorphological variations in Poecilia reticulate (Actinopterygii, Cyprinodontiformes): comparing populations of distinct environments. Iheringia, Série Zoologia, v. 105, n. 2, p. 217-222, 2015.

MONTGOMERY, D. R. Process domains and the river continuum. Journal of the American Water Resources Association, v. 35, n. 2, p. 397-410, 1999.

MUGNAI, R.; NESSIMIAN, J. L.; BAPTISTA, D. F. Manual de identificação de macroinvertebrados aquáticos do Estado do Rio de Janeiro. Rio de Janeiro: Technical Books, 2010.

MUSTOW, S. E. Biological monitoring of rivers in Thailand: use and adaptation of the BMWP score. Hydrobiologia, v. 479, n. 3, p. 191-229, 2002.

ODUM, E. P. Ecologia. Rio de Janeiro: Guanabara Koogan, 1988.

RIBEIRO, L. O.; UIEDA, V. S. Estrutura da comunidade de macroinvertebrados bentônicos de um riacho de Serra em Itatinga, São Paulo, Brasil. Revista Brasileira de Zoologia, v. 22, n. 3, p. 613-618, 2005.

RODRIGUES, A. S. L.; MALAFAIA, G.; CASTRO, P. T. A importância da avaliação do habitat no monitoramento da qualidade dos recursos hídricos: uma revisão. SaBios: Revista de Saúde e Biologia, v. 5, n. 1, p. 26-42, 2010. 
RODRÍGUEZ, M. A.; LEWIS, W. M. JR. Regulation and stability in fish assemblages of neotropical floodplain lakes. Oecologica, v. 99, p. 166-180, 1994.

SHEPP, D. L.; CUMMINS, J. D. Restoration in an urban watershed: Anacostia River of Maryland and the district of Columbia. In: WILLIAMS, J. E.; WOOD, C. A.; DOMBECK, M. P. (Ed.). Water shed restoration: principles and practices. Bethesda: American Fisheries Society, 1997.

SOUZA, F.; ABREU, J. A. S.; SILVA, C. E.; GOUVEIA, A. A. Relação entre parâmetros ecológicos e qualidade ambiental em três córregos na bacia do alto rio Paraná. Biotemas, v. 26, n. 4, p. 101-110, 2013.

SOUZA, F.; OLIVEIRA, E. F.; PAGOTTO, J. P. A.; SILVA, C. E. Estrutura ictiofaunística na zona litorânea de um lago urbano na bacia do rio Ivaí. Revista em Agronegócios e Meio Ambiente, v. 8, n. 1, p. 95-110, 2015.

SOUZA, F.; SANTOS, C. J.; TRAMONTE, R. P.; KLEPKA, V. Estrutura da comunidade de macroinvertebrados em três córregos na bacia do alto rio Paraná: uma relação entre qualidade ambiental e parâmetros ecológicos. Revista em Agronegócios e Meio Ambiente, v. 7, n. 2, p. 413-427, 2014.

SOUZA, F.; TOZZO, R. A. Poecilia reticulata Peters 1859 (Cyprinodontiformes, Poeciliidae) como possível bioindicador de ambientes degradados. Revista Meio Ambiente e Sustentabilidade, v. 3, n. 2, p. 163-175, 2013.

STATSOFT. Inc. Statistica (data analysis software system), version 7.1. 2005.

TATE, C. M.; HEINY, J. S. The ordination of benthic invertebrate communities in the South Platte River Basin in relation to environmental factors. Freshwater Biology, v. 33, n. 1, p. 439-454, 1995.

THORNE, R. J.; WILLIAMS, W. P. The response of benthic macroinvertebrates to pollution in developing countries: a multimetric system of bioassessment. Freshwater Biology, v. 37, p. 671-686, 1997.

USSEGLIO-POLATERA, P.; BOURNAUD, M.; RICHOUX, P.; TACHET, H. Biomonitoringh rough biological traits of benthic macroinvertebrates: how to use 
species trait databases? Hydrobiologia, v. 422/423, n. 1, p. 153-162, 2000.

WARD, J. V.; STANFORD, J. A. The serial discontinuity concept in lotic ecosystems. In: FONTAINE, T. D.; BARTHELL, S. M., eds. Dynamics of Lotic Ecosytems. Michigan: Ann ArborScien. Publ., p. 347-356, 1983.

YOSHIDA, C. E.; ROLLA, A. P. P. R. Ecological attributes of the benthic community and indices of water quality in urban, rural and preserved environments. Acta Limnologica Brasiliensia, v. 24, n. 3, p. 235-243, 2012.

Recebido em: 13/06/2017

Aceito em: 06/06/2018 